\title{
Network Structure, Metadata, and the Prediction of Missing Nodes and Annotations
}

\author{
Darko Hric* \\ Department of Computer Science, Aalto University School of Science, \\ P.O. Box 12200, FI-00076 Aalto, Finland
}

Tiago P. Peixoto

Department of Mathematical Sciences and Centre for Networks and Collective Behaviour, University of Bath, Claverton Down, Bath BA2 7AY, United Kingdom;

ISI Foundation, Via Alassio 11/c, 10126 Turin, Italy; and Institut für Theoretische Physik, Universität Bremen, Hochschulring 18, D-28359 Bremen, Germany

\author{
Santo Fortunato \\ Department of Computer Science, Aalto University School of Science, \\ P.O. Box 12200, FI-00076 Aalto, Finland
}

and Center for Complex Networks and Systems Research, School of Informatics and Computing, Indiana University, 47408 Bloomington, USA

(Received 7 April 2016; revised manuscript received 4 July 2016; published 12 September 2016)

\begin{abstract}
The empirical validation of community detection methods is often based on available annotations on the nodes that serve as putative indicators of the large-scale network structure. Most often, the suitability of the annotations as topological descriptors itself is not assessed, and without this it is not possible to ultimately distinguish between actual shortcomings of the community detection algorithms, on one hand, and the incompleteness, inaccuracy, or structured nature of the data annotations themselves, on the other. In this work, we present a principled method to access both aspects simultaneously. We construct a joint generative model for the data and metadata, and a nonparametric Bayesian framework to infer its parameters from annotated data sets. We assess the quality of the metadata not according to their direct alignment with the network communities, but rather in their capacity to predict the placement of edges in the network. We also show how this feature can be used to predict the connections to missing nodes when only the metadata are available, as well as predicting missing metadata. By investigating a wide range of data sets, we show that while there are seldom exact agreements between metadata tokens and the inferred data groups, the metadata are often informative of the network structure nevertheless, and can improve the prediction of missing nodes. This shows that the method uncovers meaningful patterns in both the data and metadata, without requiring or expecting a perfect agreement between the two.
\end{abstract}

DOI: 10.1103/PhysRevX.6.031038

Subject Areas: Complex Systems, Statistical Physics

\section{INTRODUCTION}

The network structure of complex systems determines their function and serves as evidence for the evolutionary mechanisms that lie behind them. However, very often their large-scale properties are not directly accessible from the network data and need to be indirectly derived via nontrivial methods. The most prominent example of this is the task of identifying modules or "communities" in networks, which has driven a substantial volume of research in recent

\footnotetext{
*darko.hric@aalto.fi

†.peixoto@bath.ac.uk

*santo@indiana.edu
}

Published by the American Physical Society under the terms of the Creative Commons Attribution 3.0 License. Further distribution of this work must maintain attribution to the author(s) and the published article's title, journal citation, and DOI. years [1-3]. Despite these efforts, it is still an open problem both how to characterize such large-scale structures and how to effectively detect them in real systems. In order to assist in bridging this gap, many researchers have compared the features extracted from such methods with known information - metadata, or "ground truth" - that putatively corresponds to the main indicators of large-scale structure [4-6]. However, this assumption is often accepted at face value, even when such metadata may contain a considerable amount of noise, are incomplete, or are simply irrelevant to the network structure. Because of this, it is not yet understood if the discrepancy observed between the metadata and the results obtained with community detection methods $[4,7]$ is mainly due to the ineffectiveness of such methods or to the lack of correlation between the metadata and actual structure.

In this work, we present a principled approach to address this issue. The central stance we take is to make no 
fundamental distinction between data and metadata and construct generative processes that account for both simultaneously. By inferring this joint model from the data and metadata, we are able to precisely quantify the extent to which the data annotations are related to the network structure, and vice versa. (Here, we consider exclusively annotation on the nodes. Networks may also possess annotations on the edges, which may be treated as edge covariates or layers, as already considered extensively in the literature; see, e.g., Refs. [8-11].) This is different from approaches that explicitly assume that the metadata (or a portion thereof) are either exactly or approximately correlated with the best network division [12-19]. With our method, if the metadata happen to be informative on the network structure, we are able to determine how; but if no correlation exists between the two, this gets uncovered as well. Our approach is more in line with a recent method by Newman and Clauset [20], who proposed using available metadata to guide prior probabilities on the network partition, but here we introduce a framework that is more general in three important ways: Firstly, we do not assume that the metadata are present in such a way that it corresponds simply to a partition of the nodes. While the latter can be directly compared to the outcome of conventional community detection methods, or used as priors in the inference of typical generative models, the majority of data sets contain much richer metadata, where nodes are annotated multiple times, with heterogeneous annotation frequencies, such that often few nodes possess the exact same annotations. Secondly, we develop a nonparametric Bayesian inference method that requires no prior information or ad hoc parameters to be specified, such as the number of communities. And, thirdly, we are able not only to obtain the correlations between structure and annotations based on statistical evidence, but we are also capable of assessing the metadata in their power to predict the network structure, instead of simply their correlation with latent partitions. This is done by leveraging the information available in the metadata to predict missing nodes in the network. This contrasts with the more common approach of predicting missing edges [21-27], which cannot be used when entire nodes have not been observed and need to be predicted, and with other approaches to detect missing nodes, which are either heuristic in nature [28] or rely on very specific assumptions on the data-generating process [29,30]. Furthermore, our method is also capable of clustering the metadata themselves, separating them in equivalence classes according to their occurrence in the network. This clustering of the metadata is done simultaneously with the clustering of the network data itself, with both aspects aiding each other, and thus providing a full generalization of the task of community detection for annotated networks. As we show, both features allows us to distinguish informative metadata from less informative ones, with respect to the network structure, as well as to predict missing annotations.
In the following, we describe our method and illustrate its use with some examples based on real data. We then follow with a systematic analysis of many empirical data sets, focusing on the prediction of nodes from metadata alone. We show that the predictiveness of network structure from metadata is widely distributed-both across and within data sets-indicating that typical network annotations vary greatly in their connection to network structure.

\section{JOINT MODEL FOR DATA AND METADATA}

Our approach is based on a unified representation of the network data and metadata. We assume here the general case where the metadata are discrete and may be arbitrarily associated with the nodes of the network. We do so by describing the data and metadata as a single graph with two node and edge types (or layers [31,32]), as shown in Fig. 1. The first layer corresponds to the network itself (the "data"), where an edge connects two "data" nodes, with an adjacency matrix $\boldsymbol{A}$, where $A_{i j}=1$ if an edge exists between two data nodes $i$ and $j$, or $A_{i j}=0$ otherwise. This layer would correspond to the entire data if the metadata were to be ignored. In the second layer both the data and the metadata nodes are present, and the connection between them is represented by a bipartite adjacency matrix $\boldsymbol{T}$, where $T_{i j}=1$ if node $i$ is annotated with a metadata token $j$ (henceforth called a tag node), or $T_{i j}=0$ otherwise. Therefore, a single data node can be associated with zero, one, or multiple tags, and likewise a single tag node may be associated with zero, one, or multiple data nodes. Within this general representation we can account for a wide

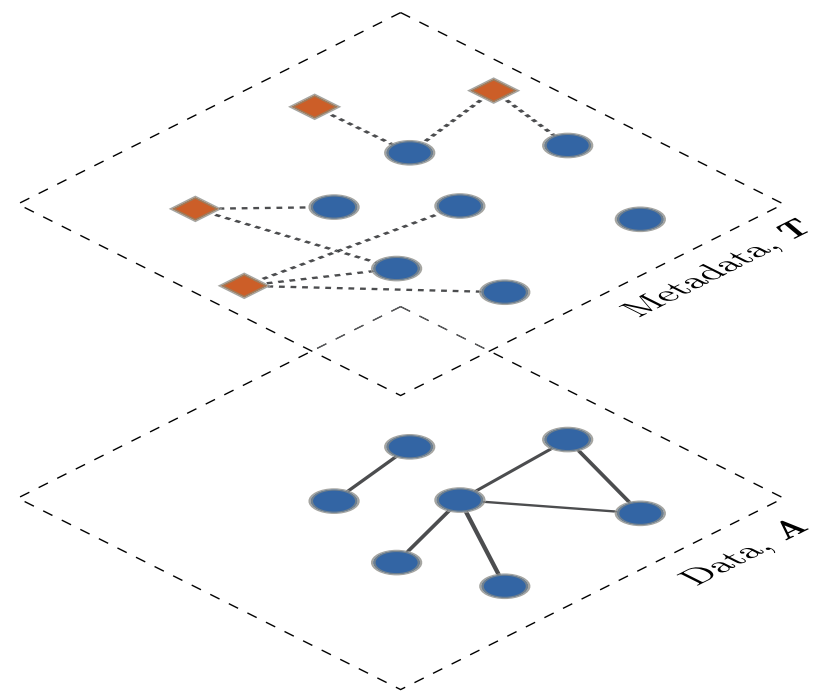

FIG. 1. Schematic representation of the joint data-metadata model. The data layer is composed of data nodes and is described by an adjacency matrix $\boldsymbol{A}$, and the metadata layer is composed of the same data nodes, as well as tag nodes, and is described by a bipartite adjacency matrix $\boldsymbol{T}$. Both layers are generated by two coupled degree-corrected SBMs, where the partition of the data nodes into groups is the same in both layers. 
spectrum of discrete node annotations. In particular, as it will become clearer below, we make no assumption that individual metadata tags actually correspond to specific disjoint groups of nodes.

We construct a generative model for the matrices $\boldsymbol{A}$ and $\boldsymbol{T}$ by generalizing the hierarchical stochastic block model (SBM) [33] with degree correction [34] for the case with edge layers [8]. In this model, the nodes and tags are divided into $B_{d}$ and $B_{t}$ groups, respectively. The number of edges between data groups $r$ and $s$ is given by the parameters $e_{r s}$ (or twice that for $r=s$ ), and between data group $r$ and tag group $u$ by $m_{r u}$. Both data and tag nodes possess fixed degree sequences, $\left\{k_{i}\right\}$ and $\left\{d_{i}\right\}$, for the data and metadata layers, respectively, corresponding to an additional set of parameters. Given these constraints, a graph is generated by placing the edges randomly in both layers independently, with a joint likelihood,

$$
P(\boldsymbol{A}, \boldsymbol{T} \mid \boldsymbol{b}, \theta, \boldsymbol{c}, \gamma)=P(\boldsymbol{A} \mid \boldsymbol{b}, \theta) P(\boldsymbol{T} \mid \boldsymbol{b}, \boldsymbol{c}, \gamma),
$$

where $\boldsymbol{b}=\left\{b_{i}\right\}$ and $\boldsymbol{c}=\left\{c_{i}\right\}$ are the group memberships of the data and tag nodes, respectively, and both $\theta=$ $\left(\left\{e_{r s}\right\},\left\{k_{i}\right\}\right)$ and $\gamma=\left(\left\{m_{r u}\right\},\left\{d_{i}\right\}\right)$ are shorthand notations for the remaining model parameters in both layers. Inside each layer, the log-likelihood is given in Eq. (2) [34,35]. [Equation (2) is an approximation that is valid for sparse graphs, where the occurrence of parallel edges can be neglected. If this is not the case, the likelihood should be appropriately modified. See Refs. [35,36] for more details.]

$$
\ln P(\boldsymbol{A} \mid \boldsymbol{b}, \theta) \approx-E-\frac{1}{2} \sum_{r s} e_{r s} \ln \frac{e_{r s}}{e_{r} e_{s}}-\sum_{i} \ln k_{i} !,
$$

and analogously for $P(\boldsymbol{T} \mid \boldsymbol{b}, \boldsymbol{c}, \gamma)$. Since the data nodes have the same group memberships in both layers, this provides a coupling between them, and we have thus a joint model for data and metadata. This model is general, since it is able to account simultaneously for the situation where there is a perfect correspondence between data and metadata (for example, when $B_{d}=B_{t}$ and the matrix $m_{r u}$ connects one data group to only one metadata group), when the correspondence is nonexistent (the matrix $\boldsymbol{T}$ is completely random, with $B_{t}=1$ ), as well as any elaborate relationship between data and metadata in between. In principle, we could fit the above model by finding the model parameters that maximize the likelihood in Eq. (1). Doing so would uncover the precise relationship between data and metadata under the very general assumptions taken here. However, for this approach to work, we need to know a priori the number of groups $B_{d}$ and $B_{t}$. This is because the likelihood of Eq. (1) is parametric (i.e., it depends on the particular choices of $\boldsymbol{b}, \boldsymbol{c}, \theta$, and $\gamma$ ), and the degrees of freedom in the model will increase with $B_{d}$ and $B_{t}$. As the degrees of freedom increase, so will the likelihood and the perceived quality of fit of the model. If we follow this criterion blindly, we will put each node and metadata tag in their individual groups, and our matrices $e_{r s}$ and $m_{r s}$ will correspond exactly to the adjacency matrices $\boldsymbol{A}$ and $\boldsymbol{T}$, respectively. This is an extreme case of overfitting, where we are not able to differentiate random fluctuations in data from actual structure that should be described by the model. The proper way to proceed in this situation is to make the model nonparametric, by including noninformative Bayesian priors on the model parameters $P(\boldsymbol{b}), P(\boldsymbol{c})$, $P(\theta)$, and $P(\gamma)$, as described in Refs. [33,36] (see also Appendix A). By maximizing the joint nonparametric likelihood, $\quad P(\boldsymbol{A}, \boldsymbol{T}, \boldsymbol{b}, \theta, \boldsymbol{c}, \gamma)=P(\boldsymbol{A}, \boldsymbol{T} \mid \boldsymbol{b}, \theta, \boldsymbol{c}, \gamma) P(\boldsymbol{b}) \times$ $P(\theta) P(\boldsymbol{c}) P(\gamma)$, we can find the best partition of the nodes and tags into groups, together with the number of groups themselves, without overfitting. This happens because, in this setting, the degrees of freedom of the model are themselves sampled from a distribution, which will intrinsically ascribe higher probabilities to simpler models, effectively working as a penalty on more complex ones. An equivalent way of justifying this is to observe that the joint likelihood can be expressed as $P(\boldsymbol{A}, \boldsymbol{T}, \boldsymbol{b}, \theta, \boldsymbol{c}, \gamma)=2^{-\Sigma}$, where $\Sigma$ is the description length of the data, corresponding to the number of bits necessary to encode both the data according to the model parameters as well as the model parameters themselves. Hence, maximizing the joint Bayesian likelihood is identical to the minimum description length criterion [37,38], which is a formalization of Occam's razor, where the simplest hypothesis is selected according to the statistical evidence available.

We note that there are some caveats when selecting the priors probabilities above. In the absence of a priori knowledge, the most straightforward approach is to select flat priors that encode this, and ascribe the same probability to all possible model parameters [39]. This choice, however, incurs some limitations. In particular, it can be shown that with flat priors it is not possible to infer with the SBM a number of groups that exceeds an upper threshold that scales with $B_{\max } \sim \sqrt{N}$, where $N$ is the number of nodes in the network [40]. Additionally, flat priors are unlikely to be good models for real data, since they assume all parameter values are equally likely. This is an extreme form of randomness that encodes maximal ignorance about the model parameters. However, no data are truly sampled from such a maximally random distribution; they are more likely to be sampled from some nonrandom distribution, but with an unknown shape. An alternative, therefore, is to postpone the decision on the prior until we observe the data, by sampling the prior distribution itself from a hyperprior. Of course, in doing so, we face the same problem again when selecting the hyperprior. For the model at hand, we proceed in the following manner: Since the matrices $\left\{e_{r s}\right\}$ and $\left\{m_{r s}\right\}$ are themselves adjacency matrices of multigraphs (with $B_{d}$ and $B_{d}+B_{t}$ nodes, respectively), we sample them from another set of SBMs, and so on, following a nested hierarchy, until the trivial model with 


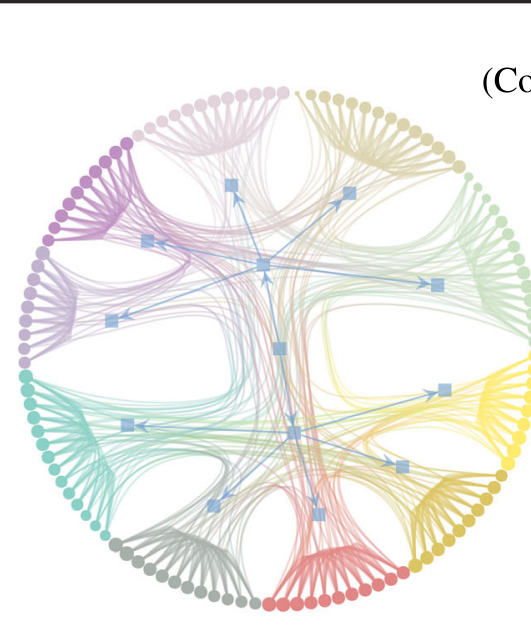

(a) Data

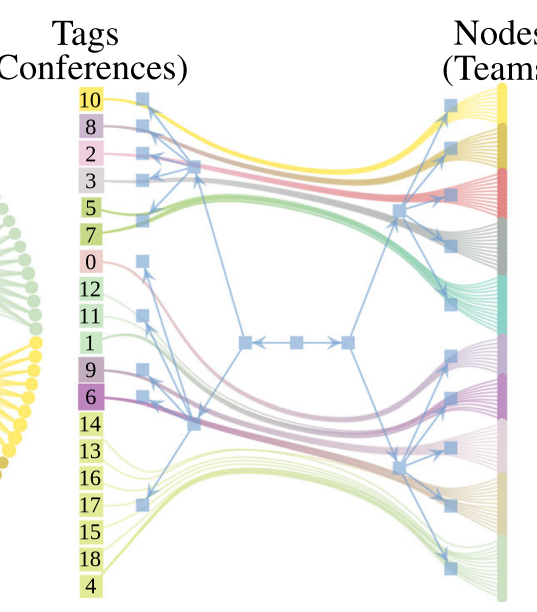

(b) Metadata

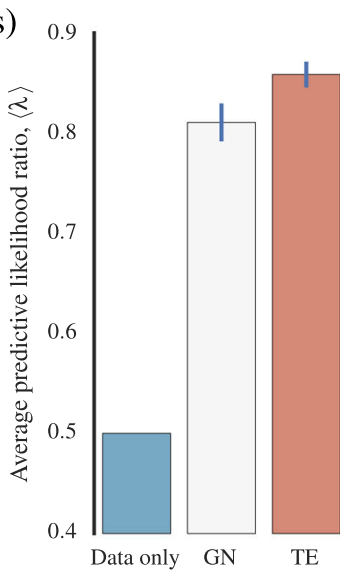

(c) Node prediction

FIG. 2. Joint data-metadata model inferred for the network of American football teams [45]. (a) Hierarchical partition of the data nodes (teams), corresponding to the "data" layer. (b) Partition of the data (teams) and tag (conference) nodes, corresponding to the second layer. (c) Average predictive likelihood of missing nodes relative to using only the data (discarding the conferences), using the original conference assignment of Ref. [45] (GN) and the corrected assignment of Ref. [46] (TE).

$B_{d}=B_{t}=1$ is reached, as described in Ref. [33]. For the remaining model parameters we select only twolevel Bayesian hierarchies, since it can be shown that higher-level ones have only negligible improvements asymptotically [36]. We review and summarize the prior probabilities in Appendix. A. With this Bayesian hierarchical model, not only do we significantly increase the resolution limit to $B_{\max } \sim N / \ln N$ [33], but also we are able to provide a description of the data at multiple scales.

It is important to emphasize that we are not restricting ourselves to purely assortative structures, as is the case in most community detection literature, but rather we are open to a much wider range of connectivity patterns that can be captured by the SBM. As mentioned in the Introduction, our approach differs from the parametric model recently introduced by Newman and Clauset [20], where it is assumed that a node can connect to only one metadata tag, and each tag is parametrized individually. In our model, a data node can possess zero, one, or more annotations, and the tags are clustered into groups. Therefore, our approach is suitable for a wider range of data annotations, where entire classes of metadata tags can be identified. Furthermore, since their approach is parametric [41], the appropriate number of groups must be known beforehand, instead of being obtained from data, which is seldom possible in practice. Additionally, when employing the fast MCMC algorithm developed in Ref. [43], the inference procedure scales linearly as $O(N)$ [or log-linearly $O\left(N \ln ^{2} N\right)$ when obtaining the full hierarchy [33] ], where $N$ is the number of nodes in the network, independently of the number of groups, in contrast to the expectationmaximization with belief propagation of Ref. [20], that scales as $O\left(B^{2} N\right)$, where $B$ is the number of groups being inferred. Hence, our method scales well not only for large networks, but also for an arbitrarily large number of communities. An implementation of our method is freely available as part of the graph-tool library [44].

This joint approach of modeling the data and metadata allows us to understand in detail the extent to which network structure and annotations are correlated, in a manner that puts neither at an advantage with respect to the other. Importantly, we do not interpret the individual tags as "ground truth" labels on the communities, and instead infer their relationships with the data communities from the entire data. Because the metadata tags themselves can be clustered into groups, we are able to assess both their individual and collective roles. For instance, if two tag nodes are assigned to the same group, this means that they are both similarly informative on the network structure, even if their target nodes are different. By following the inferred probabilities between tag and node groups, one obtains a detailed picture of their correspondence, which can deviate in principle (and often does in practice) from the commonly assumed one-to-one mapping [4,7], but includes it as a special case.

Before going into the systematic analysis of empirical data sets, we illustrate the application of this approach with a simple example, corresponding to the network of American college football teams [45], where the edges indicate that a game occurred between two teams in a given season. For these data, it is also available to which "conferences" the teams belong. Since it is expected that teams in the same conference play each other more frequently, this is assumed to be an indicator for the network structure. If we fit the above model to this data set, both the nodes (teams) and tags (conferences) are divided into $B_{d}=10$ and $B_{t}=10$ groups, respectively (Fig. 2). Some of the conferences correspond 
exactly to the inferred groups of teams, as one would expect. However, other conferences are clustered together, in particular, the independents, meaning that although they are collectively informative on the network structure, individually they do not serve as indicators of the network topology in a manner that can be conclusively distinguished from random fluctuations.

In Fig. 2, we use the conference assignments presented in Ref. [46], which are different from the original assignments in Ref. [45], due to a mistake in the original publication, where the information from the wrong season was used instead [47]. We use this as an opportunity to show how errors and noise in the metadata can be assessed with our method, while at the same time we emphasize an important application, namely, the prediction of missing nodes. We describe it in general terms, and then return to our illustration afterwards.

\section{A. Prediction of missing nodes}

To predict missing nodes, we must compute the likelihood of all edges incident on it simultaneously; i.e., for an unobserved node $i$, they correspond to the $i$ th row of the augmented adjacency matrix $\boldsymbol{a}_{i}=\left\{A_{i j}^{\prime}\right\}$, with $A_{k j}{ }^{\prime}=A_{k j}$ for $k \neq i$. If we know the group membership $b_{i}$ of the unobserved node, in addition to the observed nodes, the likelihood of the missing incident edges is

$$
\begin{aligned}
P\left(\boldsymbol{a}_{i} \mid \boldsymbol{A}, b_{i}, \boldsymbol{b}\right) & =\frac{\sum_{\theta} P\left(\boldsymbol{A}, \boldsymbol{a}_{i} \mid b_{i}, \boldsymbol{b}, \theta\right) P(\theta)}{\sum_{\theta} P(\boldsymbol{A} \mid \boldsymbol{b}, \theta) P(\theta)} \\
& =\frac{P\left(\boldsymbol{A}, \boldsymbol{a}_{i} \mid b_{i}, \boldsymbol{b}, \hat{\theta}\right) P(\hat{\theta})}{P\left(\boldsymbol{A} \mid \boldsymbol{b}, \hat{\theta}^{\prime}\right) P\left(\hat{\theta}^{\prime}\right)}
\end{aligned}
$$

where $\hat{\theta}$ and $\hat{\theta}^{\prime}$ are the only choices of parameters compatible with the node partition. However, we do not know a priori to which group the missing node belongs. If we have only the network data available (not the metadata), the only choice we have is to make the probability conditioned on the observed partition,

$$
P\left(\boldsymbol{a}_{i} \mid \boldsymbol{A}, \boldsymbol{b}\right)=\sum_{b_{i}} P\left(\boldsymbol{a}_{i} \mid \boldsymbol{A}, b_{i}, \boldsymbol{b}\right) P\left(b_{i} \mid \boldsymbol{b}\right),
$$

where $P\left(b_{i} \mid \boldsymbol{b}\right)=P\left(\boldsymbol{b}, b_{i}\right) / P(\boldsymbol{b})$. This means that we can use only the distribution of group sizes to guide the placement of the missing node, and nothing more. However, in practical scenarios we may have access to the metadata associated with the missing node. For example, in a social network we might know the social and geographical indicators (age, sex, country, etc.) of a person for whom we would like to predict unknown acquaintances. In our model, this translates to knowing the corresponding edges in the tag-node graph $\boldsymbol{T}$. In this case, we can compute the likelihood of the missing edges in the data graph as

$$
P\left(\boldsymbol{a}_{i} \mid \boldsymbol{A}, \boldsymbol{T}, \boldsymbol{b}, \boldsymbol{c}\right)=\sum_{b_{i}} P\left(\boldsymbol{a}_{i} \mid \boldsymbol{A}, b_{i}, \boldsymbol{b}\right) P\left(b_{i} \mid \boldsymbol{T}, \boldsymbol{b}, \boldsymbol{c}\right),
$$

where the node membership distribution is weighted by the information available in the full tag-node graph,

$$
\begin{gathered}
P\left(b_{i} \mid \boldsymbol{T}, \boldsymbol{b}, \boldsymbol{c}\right)=\frac{P\left(b_{i}, \boldsymbol{b} \mid \boldsymbol{T}, \boldsymbol{c}\right)}{P(\boldsymbol{b} \mid \boldsymbol{T}, \boldsymbol{c})} \\
=\frac{\sum_{\gamma} P\left(\boldsymbol{T} \mid b_{i}, \boldsymbol{b}, \boldsymbol{c}, \gamma\right) P\left(b_{i}, \boldsymbol{b}\right) P(\gamma)}{\sum_{b_{i}^{\prime}} \sum_{\gamma} P\left(\boldsymbol{T} \mid b_{i}^{\prime}, \boldsymbol{b}, \boldsymbol{c}, \gamma\right) P\left(b_{i}^{\prime}, \boldsymbol{b}\right) P(\gamma)} \\
=\frac{P\left(\boldsymbol{T} \mid b_{i}, \boldsymbol{b}, \boldsymbol{c}, \hat{\gamma}\right) P\left(b_{i}, \boldsymbol{b}\right) P(\hat{\gamma})}{\sum_{b_{i}^{\prime}} P\left(\boldsymbol{T} \mid b_{i}^{\prime}, \boldsymbol{b}, \boldsymbol{c}, \hat{\gamma}^{\prime}\right) P\left(b_{i}^{\prime}, \boldsymbol{b}\right) P\left(\hat{\gamma}^{\prime}\right)},
\end{gathered}
$$

where again $\hat{\gamma}$ and $\hat{\gamma}^{\prime}$ are the only choices of parameters compatible with the partitions $\boldsymbol{c}$ and $\boldsymbol{b}$. If the metadata correlate well with the network structure, the above distribution should place the missing node with a larger likelihood in its correct group. In order to quantify the relative predictive improvement of the metadata information for node $i$, we compute the predictive likelihood ratio $\lambda_{i} \in[0,1]$,

$$
\lambda_{i}=\frac{P\left(\boldsymbol{a}_{i} \mid \boldsymbol{A}, \boldsymbol{T}, \boldsymbol{b}, \boldsymbol{c}\right)}{P\left(\boldsymbol{a}_{i} \mid \boldsymbol{A}, \boldsymbol{T}, \boldsymbol{b}, \boldsymbol{c}\right)+P\left(\boldsymbol{a}_{i} \mid \boldsymbol{A}, \boldsymbol{b}\right)},
$$

which should take on values $\lambda_{i}>1 / 2$ if the metadata improve the prediction task, or $\lambda_{i}<1 / 2$ if they deteriorate it. The latter can occur if the metadata mislead the placement of the node (we discuss below the circumstances where this can occur).

In order to illustrate this approach we return to the American football data, and compare the original and corrected conference assignments in their capacity of predicting missing nodes. We do so by removing a node from the network, inferring the model on the modified data, and computing its likelihood according to Eqs. (5) and (7), which we use to compute the average predictive likelihood ratio for all nodes in the network, $\langle\lambda\rangle=\sum_{i} \lambda_{i} / N$. As can be seen in Fig. 2(c), including the metadata improves the prediction significantly, and indeed we observe that the corrected metadata noticeably improve the prediction when compared to the original inaccurate metadata. In short, knowing to which conference a football team belongs does indeed increase our chances of predicting against which other teams it will play, and we may do so with a higher success rate using the current conference assignments, rather than using those of a previous year. These are hardly surprising facts in this illustrative context, but the situation becomes quickly less intuitive for data sets with hundreds of thousands of nodes and a comparable number of metadata tags, for which only automated methods such as ours can be relied upon. 


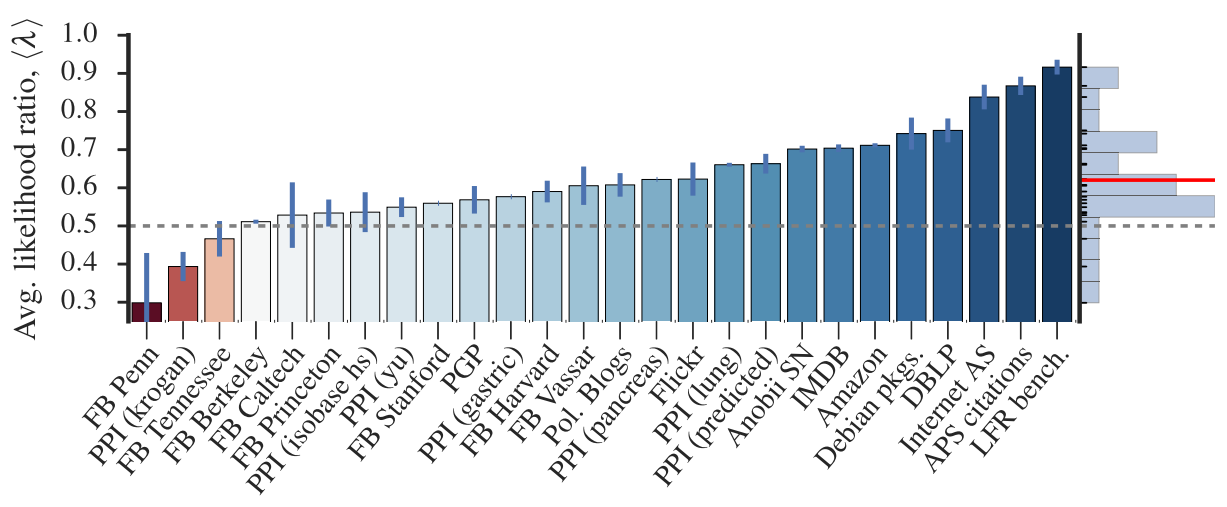

FIG. 3. Node prediction performance, measured by the average predictive likelihood ratio $\langle\lambda\rangle$ for a variety of annotated data sets (see Appendix B for descriptions). Values above 1/2 indicate that the metadata improve the node prediction task. On the right-hand axis a histogram of the likelihood ratios is shown, with a red line marking the average.

\section{EMPIRICAL DATA SETS}

We perform a survey of several network data sets with metadata (described in detail in Appendix B), where we remove a small random fraction of annotated nodes ( $1 \%$ or 100 nodes, whichever is smaller) many times, and compute the likelihood ratio $\lambda_{i}$ above for every removed node. The average value for each data set is shown in Fig. 3. We observe that for the majority of data sets the metadata are capable of improving the prediction of missing nodes, with the quality of the improvement being relatively broadly distributed. While this means that there is a positive and statistically significant correlation between the metadata and the network structure, for some data sets this leads only to moderate predictive improvements. On the other hand, there is a minority of cases where the inclusion of metadata worsens the prediction task, leading to $\langle\lambda\rangle<1 / 2$. In such situations, the metadata seem to divide the network in a manner that is largely orthogonal to the how the network itself is connected. In order to illustrate this, we consider some artificially generated data sets as follows, before returning to the empirical data sets.

\section{A. Alignment between data and metadata}

We construct a network with $N$ nodes divided into $B_{d}$ equal-sized groups that are perfectly assortative; i.e., nodes of one group are connected only to other nodes of the same group. Furthermore, the $E$ edges of the network are randomly distributed among the groups, so that they have on average the same edge density. This yields a simple structure composed of the union of $B_{d}$ disjoint, fully random networks of similar density.

In the metadata layer we have the same number of $M=N$ metadata tags, which are themselves also divided into an equal number $B_{t}=B_{d}=B$ of equal-sized groups.

In order to place $E_{m}=E$ edges between data and metadata, we also consider an alternative partition $\left\{b_{i}^{\prime}\right\}$ of the data nodes into $B$ groups that is not equal to the original partition $\left\{b_{i}\right\}$ used to construct the network. A tag in one metadata group can only connect randomly to nodes of one particular data group, and vice versa. That is, there is a one-to-one mapping between tag and data groups.

In total, we consider three ways to connect the data with the metadata:

(1) aligned with the original data partition $\left\{b_{i}\right\}$ : i.e., tagnode edges connect to the same data groups used to place the node-node edges;

(2) misaligned with the data partition: i.e., tag-node edges connect to the groups of the alternative data partition $\left\{b_{i}^{\prime}\right\}$;

(3) random: the tag-node edges are placed entirely at random, i.e., respecting neither the tag nor the node partitions.

We emphasize that 2 (misaligned) and 3 (random) are different: the former corresponds to structured metadata that are uncorrelated with the network structure, and the latter corresponds to unstructured metadata. In other words, in the misaligned case the node-tag graph is not fully random, since it connects only specific tag groups to specific node groups, whereas in the random case the node-tag edges are indeed fully random. An example of each type of construction for $B=2$ is shown in Fig. 4 .

When performing node prediction for artificial networks constructed in this manner, one observes improved prediction with aligned metadata systematically; however, with misaligned metadata a measurable degradation can be seen, while for random metadata neutral values close to $\langle\lambda\rangle=1 / 2$ are observed (see Fig. 4). The degradation observed for misaligned metadata is due to the subdivision of the data groups into $B$ smaller subgroups, according to how they are connected to the metadata tags. This subdivision, however, is not a meaningful way of capturing the pattern of the node-node connections, since all nodes that belong to the same planted group are statistically indistinguishable. If the number of subgroups is sufficiently large, this will invariably induce the incorporation of noise into the model via the different number of edges incident on each subgroup. (Note that this incorporation of noise is not 


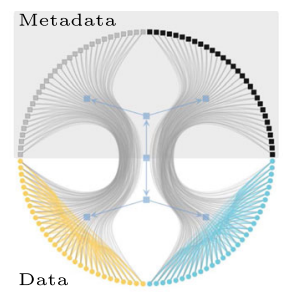

Aligned

$\widehat{2} 1.0$

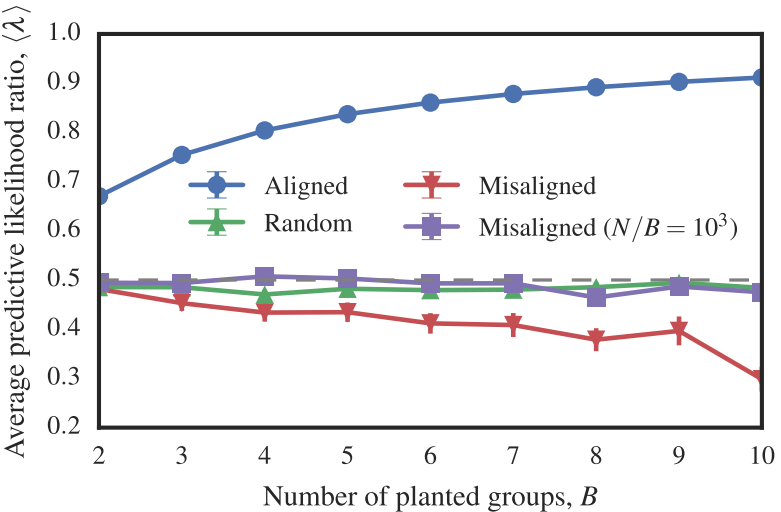

FIG. 4. Top: Examples of artificial annotated networks, showing aligned, misaligned, and random metadata, as described in the text. Bottom: Node prediction performance, measured by the likelihood ratio $\langle\lambda\rangle$, average over all possible single-node removals, for annotated networks generated with $B_{d}=B_{t}=B$ groups, $N=M=30 B$ nodes and tags, $E=E_{m}=5 N$ node-node and tag-node edges, with specific network construction given by the legend. One of the curves corresponds to networks with misaligned metadata with a larger number of nodes, $N=M=10^{3} \times B$.

strictly an overfitting, since the subdivisions are still required to properly describe the data-metadata edges.) Since these differences result only from statistical fluctuations, they are bad predictors of unobserved data, and, hence, cause the degradation in predictive quality. We note, however, that in the limiting case where the number of nodes inside each subdivision becomes sufficiently large, the degradation vanishes, since these statistical fluctuations become increasingly less relevant (see Fig. 4, curve $N / B=10^{3}$ ). Nevertheless, for sufficiently misaligned metadata, the total number of inferred data groups can increase significantly as $B_{d}=B_{d}^{0} \times B_{t}$, where $B_{d}^{0}$ is the number of data groups used to generate the network. Therefore, in practical scenarios, the presence of structured (i.e., nonrandom) metadata that are strongly uncorrelated with the network structure can indeed deteriorate node prediction, as observed in a few of the empirical examples shown in Fig. 3.

\section{B. How informative are individual tags?}

The average likelihood ratio $\langle\lambda\rangle$ used above is measured by removing nodes from the network, and include the simultaneous contribution of all metadata tags that annotate them. However, our model also divides the metadata tags into classes, which allows us to identify the predictiveness of each tag individually according to this classification. With this, one can separate informative from noninformative tags within a single data set.

We again quantify the predictiveness of a metadata tag in its capacity to predict which other nodes will connect to the one it annotates. According to our model, the probability of some data node $i$ being annotated by tag $t$ is given by

$$
P_{m}^{t}(i \mid t)=d_{i} \frac{m_{b_{i}, c_{t}}}{m_{b_{i}} m_{c_{t}}},
$$

which is conditioned on the group memberships of both data and metadata nodes. Analogously, the probability of some data node $i$ being a neighbor of a chosen data node $j$ is given by

$$
P_{e}(i \mid j)=k_{i} \frac{e_{b_{i}, b_{j}}}{e_{b_{i}} e_{b_{j}}}
$$

Hence, the probability of $i$ being a neighbor of any node $j$ that is annotated with tag $t$ is given by

$$
P_{t}(i)=\sum_{j} P(i \mid j) P_{m}(j \mid t) .
$$

In order to compare the predictive quality of this distribution, we need to compare it to a null distribution where the tags connect randomly to the nodes,

$$
Q(i)=\sum_{j} P(i \mid j) \Pi(j),
$$

where $\Pi(i)=d_{i} / M$, with $M=\sum_{r<s} m_{r s}$, is the probability that node $i$ is annotated with any tag at random. The information gain obtained with the annotation is then quantified by the Kullback-Leibler divergence between both distributions:

$$
D_{\mathrm{KL}}\left(P_{t} \| Q\right)=\sum_{i} P_{t}(i) \ln \frac{P_{t}(i)}{Q(i)} .
$$

This quantity measures the amount of information lost when we use the random distribution $Q$ instead of the metadata-informed $P_{t}$ to characterize possible neighbors, and, hence, the amount we gain when we do the opposite. It is a strictly positive quantity that can take any value between zero and $-\ln Q^{*}$, where $Q^{*}$ is the smallest nonzero value of $Q(i)$. If we substitute Eqs. (12) and (11) in Eq. (15), we notice that it depends on only the group membership of $t$, and can be written as

$$
D_{\mathrm{KL}}\left(P_{t} \| Q\right)=D_{\mathrm{KL}}\left(p_{c_{t}} \| q\right)
$$

with 


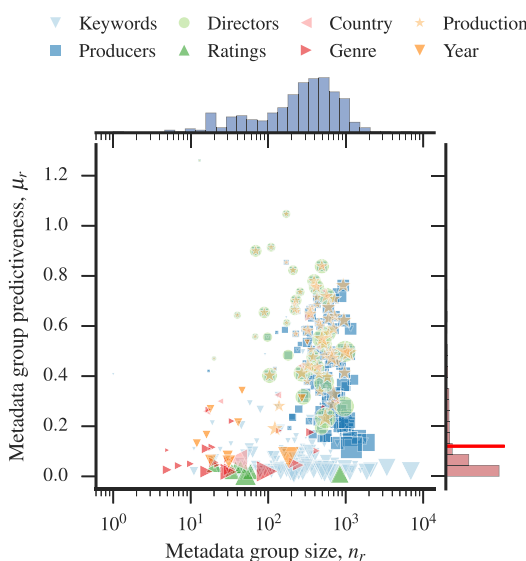

(a) IMDB

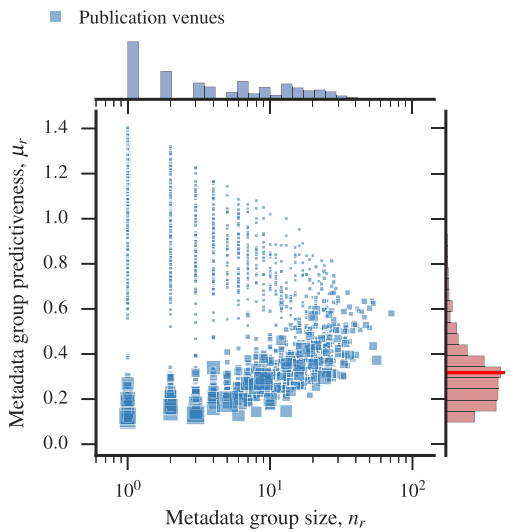

(d) DBLP

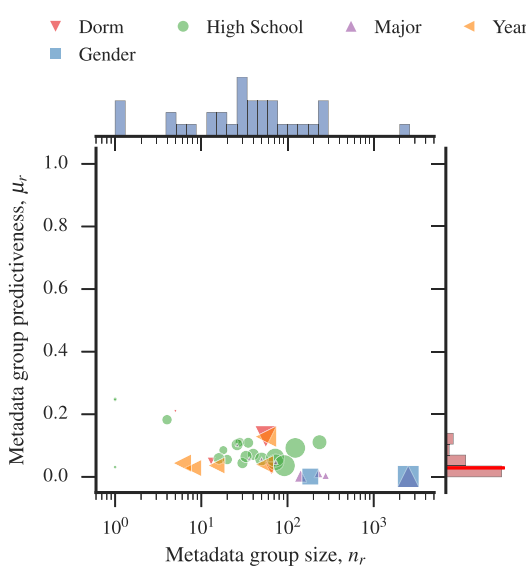

(g) FB Penn

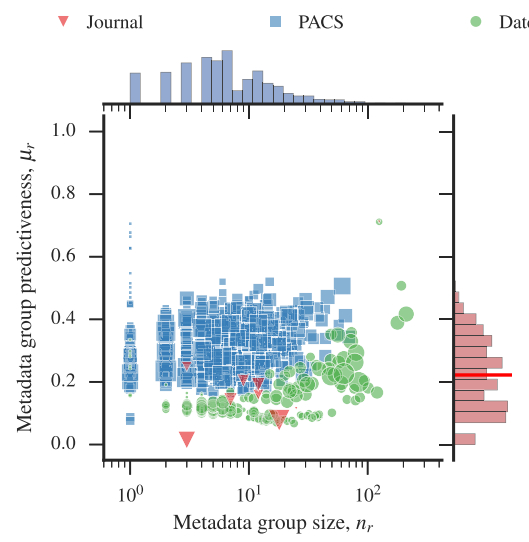

(b) APS citations

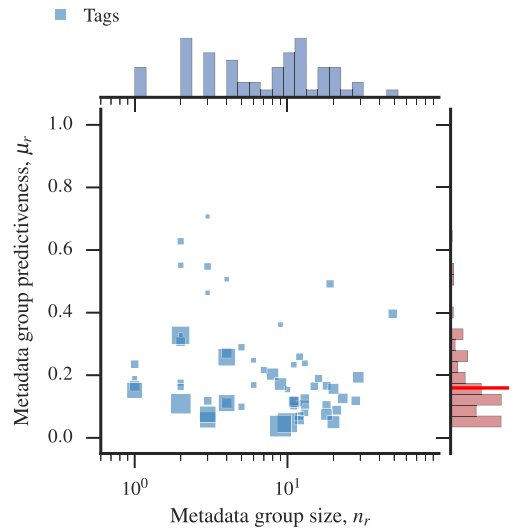

(e) Debian packages

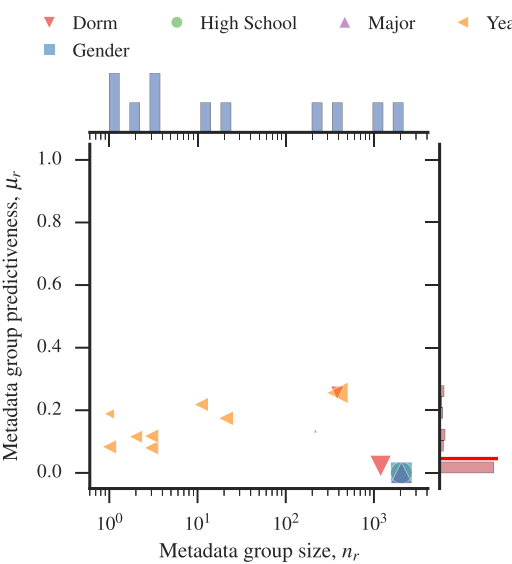

(h) FB Harvard

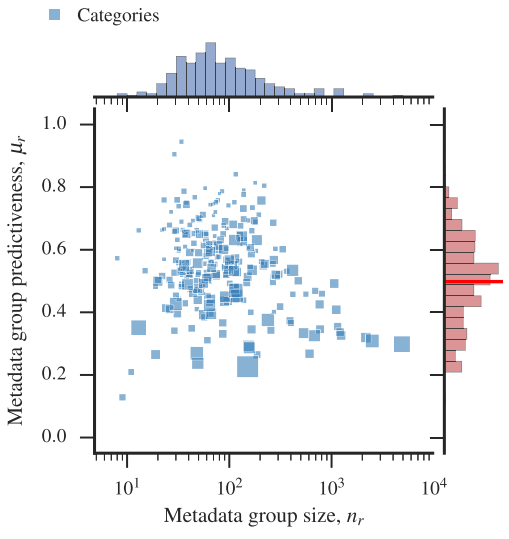

(c) Amazon

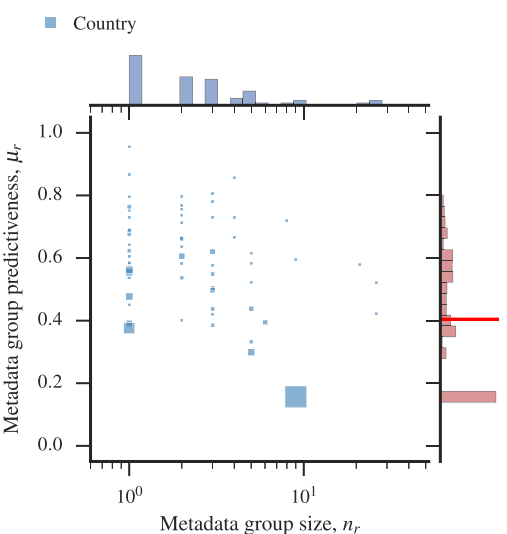

(f) Internet AS

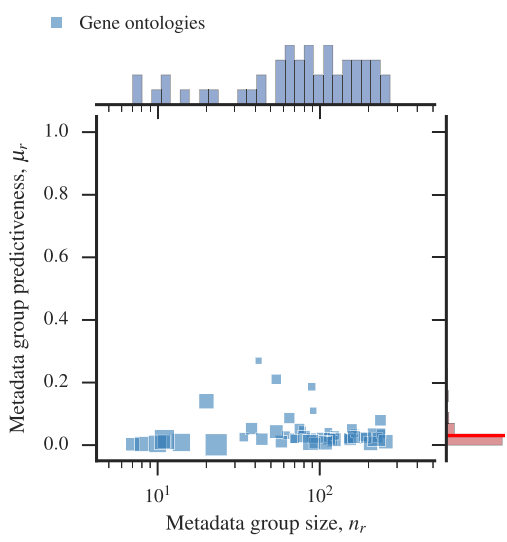

(i) PPI (Krogan)

FIG. 5. Metadata predictiveness for several empirical data sets. The panels show the predictiveness of metadata groups $\mu_{r}$ [Eq. (18)] versus metadata group sizes $n_{r}$. The sizes of the symbols indicate the metadata frequency. The symbols correspond to the most frequent types of tags in each group (which may contain tags of different types). On the axis of each figure are shown marginal histograms, weighted according to the tag frequencies. A red horizontal line marks the average predictiveness.

$$
p_{r}(u)=\sum_{s} p_{e}(u \mid s) p_{m}(s \mid u), \quad q(u)=\sum_{s} p_{e}(u \mid s) \pi(s),
$$

being the probabilities of a node that belongs to group $u$ being a neighbor of a node annotated by a tag belonging to group $r$, for both the structured and random cases, where $p_{e}(u \mid s)=e_{u s} / e_{s}, p_{m}(s \mid u)=m_{s r} / m_{r}$, and $\pi(s)=m_{s} / M$. Since this can take any value between zero and $-\ln q^{*}$, where $q^{*}$ is the smallest nonzero value of $q(u)$, this will, in general, depend on how many edges there are in the 
network, given that $q^{*} \geq 1 / 2 E$. For a concise comparison between data sets of different sizes, it is useful to consider a relative version of this measure that does not depend on the size. Although one option is to normalize by the maximum possible value, here we use instead the entropy of $q$, $H(q)=-\sum_{r} q(r) \ln q(r)$, and denote the predictiveness $\mu_{r}$ of tag group $r$ as

$$
\mu_{r} \equiv \frac{D_{\mathrm{KL}}\left(p_{r} \| q\right)}{H(q)}
$$

This gives us the relative improvement of the annotated prediction with respect to the uniformed one. Although it is possible to have $\mu_{r}>1$, this is not typical even for highly informative tags, and would mean that a particularly unlikely set of neighbors becomes particularly likely once we consider the annotation. Instead, a more typical highly informative metadata annotation simply narrows down the predicted neighborhood to a typical group sampled from $q$.

Using the above criterion, we investigate in detail the data sets of Fig. 3 and quantify the predictiveness of the node annotations, as is shown in Fig. 5 for a selected subset. Overall, we observe that the data sets differ greatly not only in the overall predictiveness of their annotations, but also in the internal structures. Typically, we find that within a single data set the metadata predictiveness is widely distributed. A good example of this is the IMDB data, which describe the connection between actors and films, and include annotations on the films corresponding to the year and country of production, the producers, the production company, the genres, user ratings, as well as usercontributed keywords. In Fig. 5(a), we see that the larger fraction of annotations posses very low predictiveness (which includes the vast majority of user-contributed keywords and ratings); however, there is still a significant number of annotations that can be quite predictive. The most predictive types of metadata are combinations of producers and directors (e.g., Cartoon productions), followed by specific countries (e.g., New Zealand, Norway), and year of productions. Besides keywords and ratings, film genres are among those with the lowest predictiveness. A somewhat narrower variability is observed for the APS citation data in Fig. 5(b), where the three types of annotations are clearly distinct. The PACS numbers are the most informative on average, followed by the date of publication (with older dates being more predictive then new ones-presumably due to the increasing publication volume and diversification over the years), and lastly the journal. One prominent exception is the most predictive metadata group that corresponds to the now-extinct Physical Review (Series I) journal, and its publication dates ranging from 1893 to 1913. For the Amazon data set of Fig. 5(c), the metadata also exhibit significant predictive variance, but there are no groups of tags that possess very low values, indicating that most product categories are indeed strong indications of co-purchases. This is similar to what is observed for the internet AS, with most countries being good predictors of the network structure. The least predictive annotations happen to be a group of ten countries that include the U.S. as the most frequent one. A much wider variance is observed in the DBLP collaboration network, where the publication venues seem to be divided into two branches: very frequent and popular ones with low to moderate predictiveness, and many very infrequent ones with high to very high predictiveness. For other data sets a wide variance in predictiveness is not observed. In particular, for most Facebook networks as well as proteinprotein interaction networks, the available metadata seem to be only tenuously correlated with the network structure, with narrowly distributed values of low predictiveness, in accordance with their relatively low placement in Fig. 3.

\section{PREDICTION OF MISSING METADATA}

Since we have defined a full joint model for data and metadata, our framework is not restricted to prediction of missing nodes, but can also predict missing edges both in the data and metadata layers. The latter can be used to predict incomplete metadata information, which corresponds to missing edges between data nodes and metadata tags, as follows. Suppose the tag layer is decomposed as the union of two edge sets, $\delta \boldsymbol{T} \cup \boldsymbol{T}$, where $\boldsymbol{T}$ is a set of observed data-metadata edges, and $\delta \boldsymbol{T}$ is a set of missing edges of the same type. Under our model, we can write the marginal posterior likelihood for $\delta \boldsymbol{T}$ as

$$
P(\delta \boldsymbol{T} \mid \boldsymbol{T}, \boldsymbol{b}, \boldsymbol{c})=\frac{P(\delta \boldsymbol{T} \cup \boldsymbol{T} \mid \boldsymbol{T}, \boldsymbol{b}, \boldsymbol{c})}{P(\boldsymbol{T} \mid \boldsymbol{b}, \boldsymbol{c})},
$$

where $P(\boldsymbol{T} \mid \boldsymbol{b}, \boldsymbol{c})=\sum_{\delta \boldsymbol{T}} P(\delta \boldsymbol{T} \cup \boldsymbol{T} \mid \boldsymbol{b}, \boldsymbol{c})$ is a normalization constant. If we have our set of missing edges coming from a restricted set of possibilities, $\delta \boldsymbol{T} \in\left\{\delta \boldsymbol{T}_{1}, \delta \boldsymbol{T}_{2}, \ldots\right\}$, we may write the predictive likelihood ratio

$$
\lambda_{i}=\frac{P\left(\delta \boldsymbol{T}_{i} \mid \boldsymbol{T}, \boldsymbol{b}, \boldsymbol{c}\right)}{\sum_{j} P\left(\delta \boldsymbol{T}_{j} \mid \boldsymbol{T}, \boldsymbol{b}, \boldsymbol{c}\right)}=\frac{P\left(\delta \boldsymbol{T}_{i} \cup \boldsymbol{T} \mid \boldsymbol{b}, \boldsymbol{c}\right)}{\sum_{j} P\left(\delta \boldsymbol{T}_{j} \cup \boldsymbol{T} \mid \boldsymbol{b}, \boldsymbol{c}\right)},
$$

where the normalization constant of Eq. (19) no longer plays a role. Hence, if we want to compare the likelihood of a given set of alternative node annotations, all we need to do is to infer the parameters $\boldsymbol{b}$ and $\boldsymbol{c}$ of the model given the observed network,

$$
\{\hat{\boldsymbol{b}}, \hat{\boldsymbol{c}}\}=\underset{\{\boldsymbol{b}, \boldsymbol{c}\}}{\operatorname{argmax}} P(\boldsymbol{T} \mid \boldsymbol{b}, \boldsymbol{c}) P(\boldsymbol{b}) P(\boldsymbol{c}),
$$

and then add the missing edges $\delta \boldsymbol{T}_{i}$ to the likelihood using this parameter estimate to compute the likelihood ratio of Eq. (20).

We illustrate the application of our method again with the American college football data. For each data node 

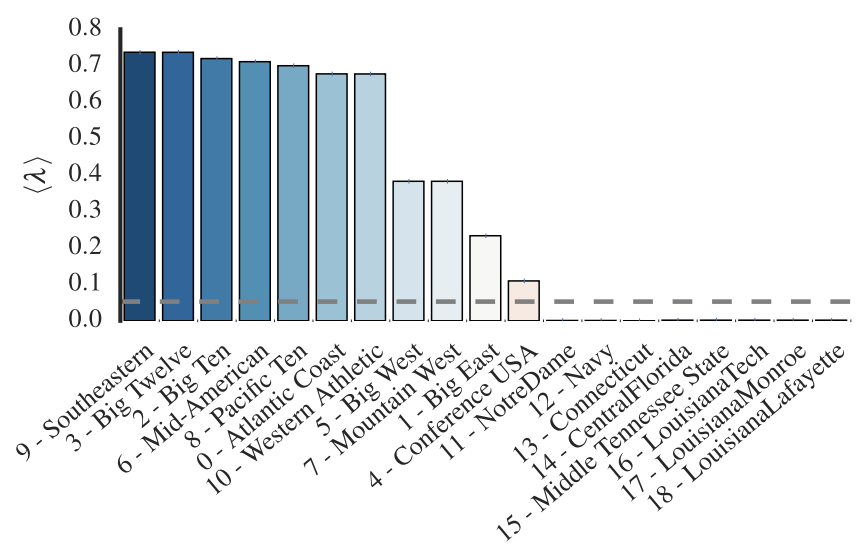

FIG. 6. Average predictive likelihood ratio $\langle\lambda\rangle$ of missing metadata tags (conferences) for the American football data, using the annotations given in Ref. [47]. Tags 11-18 are "independents," i.e., teams that do not belong to any conference. The dashed line marks the value $1 / 19$, corresponding to a uniform likelihood between all tags.

(team), we remove the single metadata tag associated with it (i.e., the team's conference), perform the model inference, and compute the predictive likelihood ratio of Eq. (20) for the removed tag, with respect to all other possible tags. The averages over all teams that belong to a given conference are shown in Fig. 6. The method succeeds in predicting the correct conference assignment with the highest likelihood in all cases, except for the "independent" teams. These teams do not belong to any conference, and are therefore assigned a unique conference tag. When this assignment is removed, it leaves an independent tag without any connection to the graph, and hence our model is not able to predict its placement. But since there is no additional information in the data once this sole assignment is removed, it is simply impossible to make an informative guess. In the cases where it is possible, our approach seems able to leverage the available information and increases the changes of successful metadata prediction.

\section{CONCLUSION}

We present a general model for the large-scale structure of annotated networks that does not intrinsically assume that there is a direct correspondence between metadata tags and the division of network into groups, or communities. Instead, we assume that the data-metadata correlation is itself generated by an underlying process, with parameters that are unknown a priori. We present a Bayesian framework to infer the model parameters from data, which is capable of uncovering - in addition to the network structure- the connection between network structure and annotations, if there is one to be found. We show how this information can be used to predict missing nodes in the network when only the annotations are known.

When applying the method for a variety of annotated data sets, we find that their annotations lie in a broad range with respect to their correlation with network structure. For most data sets considered, there is evidence for statistically significant correlations between the annotations and the network structure, in a manner that can be detected by our method, and exploited for the task of node prediction. For a few data sets, however, we find evidence of metadata which are not trivially structured, but seem to be largely uncorrelated with the actual network structure.

The predictiveness variance of metadata observed across different data sets is also often found inside individual data sets. Typically, single data sets possess a wealth of annotations, most of which are not very informative on the network structure, but a smaller fraction clearly is. Our method is capable of separating groups of annotations with respect to their predictiveness, and hence can be used to prune such data sets from "metadata noise" by excluding low-performing tags from further analysis.

As is always true when doing statistical inference, results obtained are conditioned on the validity of the model formulation, which invariably includes assumptions about the data-generating process. In our case, this means that the data-metadata layer can be represented as a graph, and that it is well modeled by a SBM. Naturally, this is only one of many possibilities, and it remains an open problem to determine which alternatives work best for any given annotated network. This is particularly true for annotations that correspond to continuous values (e.g., time and space), which either would need to be discretized before the application of our method or, preferably, would require a different modeling ansatz (see, e.g., Ref. [20]).

Nevertheless, we argue that the present approach is an appropriate starting point that provides an important but overlooked perspective in the context of community detection validation. In a recent study [7] a systematic comparison between various community detection methods and node annotations was performed, where for most of them strong discrepancies were observed. If we temporarily (and unjustifiably) assume a direct agreement with available annotations as the "gold standard," this discrepancy can be interpreted in a few ways. Firstly, the methods might be designed to find structures that fit the data poorly, and hence cannot capture their most essential features. Secondly, even if the general ansatz is sound, a given algorithm might still fail for more technical and subtle reasons. For example, most methods considered in Ref. [7] do not attempt to gauge the statistical significance of their results, and hence are subject to overfitting $[48,49]$. This incorporation of statistical noise will result in largely meaningless division of the networks, which would be poorly correlated with the "true" division. Additionally, recently Newman and Clauset [20] suggested that while the best-fitting division of the network can be poorly correlated with the metadata, the network may still admit alternative divisions that are also statistically significant, but happen to be well correlated with the annotations. 
On the other hand, the metadata heterogeneity we find with our method gives a strong indication that node annotations should not be used in direct comparisons to community detection methods in the first place-at least not indiscriminately. In most networks we analyze, even when the metadata are strongly predictive of the network structure, the agreement between the annotations and the network division tends to be complex, and very different from the one-to-one mapping that is more commonly assumed. Furthermore, almost all data sets contain considerable noise in their annotations, corresponding to metadata tags that are essentially random. From this, we argue that data annotations should not be used as a panacea in the validation of community detection methods. Instead, one should focus on validation methods that are grounded in statistical principles, and use the metadata as a source of additional evidence-possessing their own internal structures and also subject to noise, erros and ommissions-rather than a form of absolute truth.

\section{ACKNOWLEDGMENTS}

We acknowledge the computational resources provided by the Aalto Science-IT project. D. H. and S. F. gratefully acknowledge MULTIPLEX, Grant No. 317532 of the European Commission. T.P.P acknowledges support from the University of Bremen under funding program ZF04. This work was supported by the European Community's H2020 Program under the scheme "INFRAIA-1-2014-2015: Research Infrastructures", Grant agreement No. 654024 "SoBigData: Social Mining \& Big Data Ecosystem" (http://www.sobigdata.eu).

\section{APPENDIX A: MODEL LIKELIHOOD AND PRIORS}

As mentioned in the text, the microcanonical degreecorrected SBM log-likelihood is given by [35]

$$
\ln P(\boldsymbol{A} \mid \boldsymbol{b}, \theta) \approx-E-\frac{1}{2} \sum_{r s} e_{r s} \ln \frac{e_{r s}}{e_{r} e_{s}}-\sum_{i} \ln k_{i} !
$$

(if Stirling's factorial approximation is used) and likewise for $\ln P(\boldsymbol{T} \mid \boldsymbol{c}, \gamma)$, where one replaces $e_{r s}$ by $m_{r s}$ and $k_{i}$ by $d_{i}$,

$$
\ln P(\boldsymbol{T} \mid \boldsymbol{c}, \gamma) \approx-M-\frac{1}{2} \sum_{r s} m_{r s} \ln \frac{m_{r s}}{m_{r} m_{s}}-\sum_{i} \ln d_{i} !,
$$

where $E=\sum_{r s} e_{r s} / 2$ and $M=\sum_{r s} m_{r s} / 2$. This assumes that the graph is sufficiently sparse, otherwise corrections need to be introduced, as described in Ref. [35,36]. In order to compute the full joint likelihood, we need priors for the parameters $\left\{b_{i}\right\},\left\{c_{i}\right\},\left\{k_{i}\right\},\left\{d_{i}\right\},\left\{e_{r s}\right\}$, and $\left\{m_{r s}\right\}$.

For the node partitions, we use a two-level Bayesian hierarchy as done in Ref. [33], where one first samples the group sizes from a random histogram, and then the node partition randomly conditioned on the group sizes. The nonparametric likelihood is given by $P\left(\left\{b_{i}\right\}\right)=e^{-\mathcal{L}_{p}}$, with

$$
\mathcal{L}_{p}=\ln \left(\left(\begin{array}{c}
B \\
N
\end{array}\right)\right)+\ln N !-\sum_{r} \ln n_{r} !
$$

where $\left.\left(\begin{array}{c}n \\ m\end{array}\right)\right)=\left(\begin{array}{c}n+m-1 \\ m\end{array}\right)$ is the total number of $m$ combinations with repetitions from a set of size $n$. The prior $P\left(\left\{c_{i}\right\}\right)$ is analogous.

For the degree sequences, we proceed in the same fashion [36], by sampling the degrees conditioned on the total number of edges incident on each group, by first sampling a random degree histogram with a fixed average, and finally the degree sequence conditioned on this distribution. This leads to a likelihood $P\left(\left\{k_{i}\right\} \mid\left\{e_{r s}\right\},\left\{b_{i}\right\}\right)=e^{-\mathcal{L}_{\kappa}}$, with

$$
\mathcal{L}_{\kappa}=\sum_{r} \ln \Xi_{r}+\ln n_{r} !-\sum_{k} \ln n_{k}^{r} !,
$$

where $\ln \Xi_{r} \simeq 2 \sqrt{\zeta(2) e_{r}}$. Again, the likelihood for $P\left(\left\{d_{i}\right\} \mid\left\{m_{r s}\right\},\left\{c_{i}\right\}\right)$ is entirely analogous.

For the matrix of edge counts $\left\{e_{r s}\right\}$, we use the hierarchical prior proposed in Ref. [33]. Here, we view this matrix as the adjacency matrix of a multigraph with $B_{d}$ nodes and $E_{d}=\sum_{r s} e_{r s} / 2$ edges. We sample this multigraph from another SBM with a number of groups $B_{d}^{1}$, which itself is sampled from another SBM with $B_{d}^{2}$ groups, and so on, until $B_{d}^{L}=1$ for some depth $L$. The whole nonparametric likelihood is then $P\left(\left\{e_{r s}\right\}\right)=e^{-\Sigma}$, with

$$
\Sigma=\sum_{l=1}^{L} S_{m}\left(\left\{e_{r s}^{l}\right\},\left\{n_{r}^{l}\right\}\right)+\mathcal{L}_{t}^{l-1},
$$

with $\left\{e_{r s}^{l}\right\},\left\{n_{r}^{l}\right\}$ describing the block model at level $l$, and

$$
\mathcal{S}_{m}=\sum_{r>s} \ln \left(\left(\begin{array}{c}
n_{r} n_{s} \\
e_{r s}
\end{array}\right)\right)+\sum_{r} \ln \left(\left(\begin{array}{c}
\left(\left(\begin{array}{c}
n_{r} \\
2
\end{array}\right)\right) \\
e_{r r} / 2
\end{array}\right)\right),
$$

is the entropy of the corresponding multigraph ensemble and

$$
\mathcal{L}_{t}^{l}=\ln \left(\left(\begin{array}{c}
B_{l} \\
B_{l-1}
\end{array}\right)\right)+\ln B_{l-1} !-\sum_{r} \ln n_{r}^{l} !
$$

is the description length of the node partition at level $l>0$. The procedure is exactly the same for the prior $P\left(\left\{m_{r s}\right\}\right)$.

\section{APPENDIX B: DATA SETS}

Here we list descriptions of the annotated data sets used in this work. Basic statistics are given in Table I. 
TABLE I. Summary of the basic statistics of the data sets used in this work. $N_{d}$ and $E_{d}$ are the number of data nodes and data-data edges, respectively, whereas $N_{t}$ and $E_{t}$ are the number of metadata tags and node-tag edges, respectively. $B_{d}$ and $B_{t}$ are the number of data and metadata groups inferred with our method.

\begin{tabular}{|c|c|c|c|c|c|c|}
\hline Data set & $N_{d}$ & $E_{d}$ & $N_{t}$ & $E_{t}$ & $B_{d}$ & $B_{t}$ \\
\hline LFR & 1000 & 9839 & 40 & 1000 & 29 & 29 \\
\hline PPI (Krogan) & 5247 & 45899 & 4896 & 54904 & 62 & 55 \\
\hline PPI (Yu) & 964 & 1487 & 2119 & 10304 & 16 & 17 \\
\hline PPI (isobase-hs) & 8580 & 34250 & 1972 & 20633 & 40 & 15 \\
\hline PPI (gastric) & 4763 & 26131 & 10445 & 94035 & 50 & 50 \\
\hline PPI (lung) & 4843 & 27459 & 10948 & 100492 & 55 & 50 \\
\hline PPI (pancreas) & 4759 & 25978 & 10444 & 93686 & 49 & 46 \\
\hline PPI (predicted) & 7606 & 23446 & 12337 & 143847 & 69 & 68 \\
\hline FB Caltech & 762 & 16651 & 591 & 4145 & 22 & 5 \\
\hline FB Penn & 41536 & 1362220 & 4805 & 216349 & 365 & 29 \\
\hline FB Harvard & 15086 & 824595 & 3942 & 74293 & 192 & 15 \\
\hline FB Stanford & 11586 & 568309 & 3337 & 57940 & 182 & 12 \\
\hline FB Berkeley & 22900 & 852419 & 2906 & 116556 & 267 & 16 \\
\hline FB Princeton & 6575 & 293307 & 2396 & 32901 & 110 & 10 \\
\hline FB Tennessee & 16977 & 770658 & 2660 & 89458 & 271 & 20 \\
\hline FB Vassar & 3068 & 119161 & 1620 & 16859 & 69 & 12 \\
\hline Political blogs & 1222 & 16714 & 2 & 1222 & 12 & 2 \\
\hline DPD & 35029 & 161313 & 580 & 115999 & 253 & 59 \\
\hline PGP & 39796 & 197150 & 35370 & 148966 & 485 & 380 \\
\hline Internet AS & 46676 & 262953 & 225 & 45987 & 224 & 59 \\
\hline aNobii & 140687 & 869448 & 8003 & 926403 & 194 & 70 \\
\hline Amazon & 366997 & 987942 & 43807 & 1775085 & 4477 & 255 \\
\hline DBLP & 317080 & 1049866 & 13477 & 719820 & 4667 & 1746 \\
\hline IMDB & 372787 & 1812657 & 139025 & 3030003 & 843 & 328 \\
\hline APS citations & 437914 & 4596335 & 22530 & 1916281 & 5681 & 954 \\
\hline Flickr & 1624992 & 15476836 & 99270 & 8493666 & 779 & 158 \\
\hline
\end{tabular}

LFR.-Lancichinetti-Fortunato-Radicchi benchmark graph with $N=1000$ vertices and community sizes between 10 and 50, with mixing parameter $\mu=0.5$ [50]. The remaining parameters are the same as in Ref. [50]. This model corresponds to a specific parametrization of the degree-corrected SBM [34], and is often used to test and optimize most current algorithms, and thus serves as a baseline reference for a network with known and detectable structure. The network was created with standard LFR code [51].

PPI networks. - In these networks nodes are individual proteins, and there is a link between them if there is a confirmed interaction. Protein labels from Gene Ontology project (GO) [52] are used as node annotations. The networks themselves correspond to several different sources: Krogan and $Y u$ correspond to yeast (Saccharomyces cerevisiae), from two different publications: Krogan [53] and Yu [54]; isobase-hs corresponds to human proteins, as collected by the Isobase project [55]; Predicted include predicted and experimentally determined proteinprotein interactions for humans, from the PrePPI project [56] (human interactions that are in the $\mathrm{HC}$ reference set predicted by structural modeling but not nonstructural clues); Gastric, pancreas, lung are obtained by splitting the PrePPI network [56] by the tissue where each protein is expressed.
Facebook networks (FB).-Networks of social connections on the Facebook online social network, obtained in 2005, corresponding to students of different universities [57]. All friendships are present as undirected links, as well as six types of annotation: Dorm (residence hall), major, second major, graduation year, former high school, and gender.

Internet AS.-Network of the internet at the level of autonomous aystems (AS). Nodes represent autonomous systems, i.e., systems of connected routers under the control of one or more network operators with a common routing policy. Links represent observed paths of internet protocol traffic directly from one AS to another. The node annotations are countries of registration of each AS. The data are obtained from the CAIDA project [58].

DBLP. - Network of collaboration of computer scientists. Two scientists are connected if they have coauthored at least one paper [59]. Node annotations are publication venues (scientific conferences). Data downloaded from SNAP $[4,60]$.

aNobii.-This is an online social network for sharing book recommendations, popular in Italy. Nodes are user profiles, and there can be two types of directed relationships between them, which we use as undirected links ("friends" and "neighbors"). Data were provided by Aiello 
and co-workers $[61,62]$. We use all present node metadata, of which there are four kinds: age, location, country, and membership.

PGP.-The "web of trust" of PGP (pretty good privacy) key signings, representing an indication of trust of the identity of one person (signee) by another (signer). A node represents one key, usually but not always corresponding to a real person or organization. Links are signatures, which by convention are intended to be made only if the two parties are physically present, have verified each others' identities, and have verified the key fingerprints. Data are taken from a 2009 snapshot of public SKS key servers [63].

Flickr.-Picture sharing web site and social network, as crawled by Mislove et al. [64]. Nodes are users and edges exist if one user "follows" another. The node annotations are user groups centered around a certain type of content, such as "nature" or "Finland."

Political blogs. - A directed network of hyperlinks between weblogs on U.S. politics, recorded in 2005 by Adamic and Glance [65]. Links are all front-page hyperlinks at the time of the crawl. Node annotations are "liberal" or "conservative" as assigned by either blog directories or occasional self-evaluation.

Debian packages.-Software dependencies within the Debian GNU-Linux operating system [66]. Nodes are unique software packages, such as linux-image-2.6amd64, libreoffice-gtk, or python-scipy. Links are the "depends," "recommends," and "suggests" relationships, which are a feature of Debian's APT package management system designed for tracking dependencies. Node annotations are tag memberships from the DebTags project [67], such as devel::lang:python or web::browser [68]. The network was generated from package files in Debian 7.1 Wheezy as of 2013-07-15, "main" area only. Similar files are freely available in every Debian-based OS. Tags can be found in Ref. [69].

Amazon.-Network of product copurchases on online retailer Amazon. Nodes represent products, and edges are said to represent copurchases by other customers presented on the product page [70]. The true meaning of links is unknown and is some function of Amazon's recommendation algorithm. Data were scraped in mid-2006 and downloaded from Ref. [71]. We use copurchasing relationships as undirected edges. Product categories are used as node annotations. Although product categories are hierarchical by nature, we use only the end points (or "leaves") of the hierarchy: Books/Fiction/Fantasy/Epic and Books/Nonfiction are two different metadata labels.

$I M D B$. - This network is compiled by extracting information available in the internet movie database (IMDB) [72], and it contains each cast member and film as distinct nodes, and an undirected edge exists between a film and each of its cast members. The network used here corresponds to a snapshot made in 2012 [40]. The node annotations are the following information available on the films: country and year of production, production company, producers, directors, genre, user-contributed keywords, and genres.

APS citations.-This network corresponds to directed citations between papers published in journals of the American Physical Society (APS) for a period of over 100 years [73]. The node annotations correspond to PACS classification tags, journal, and publication date.

[1] S. Fortunato, Community Detection in Graphs, Phys. Rep. 486, 75 (2010).

[2] M. A. Porter, J.-P. Onnela, and P. J. Mucha, Communities in Networks, Not. Am. Math. Soc. 56, 1082 (2009).

[3] M. E. J. Newman, Communities, Modules and Large-Scale Structure in Networks, Nat. Phys. 8, 25 (2011).

[4] J. Yang and J. Leskovec, in Proceedings of the ACM SIGKDD Workshop on Mining Data Semantics, MDS '12 (ACM, New York, 2012), pp. 3:1-3:8.

[5] J. Yang and J. Leskovec, in Proceedings of the 2012 IEEE 12th International Conference on Data Mining (ICDM) (IEEE, New York, 2012), pp. 1170-1175.

[6] J. Yang and J. Leskovec, Structure and Overlaps of Communities in Networks, arXiv:1205.6228.

[7] D. Hric, R. K. Darst, and S. Fortunato, Community Detection in Networks: Structural Clusters versus Ground Truth, Phys. Rev. E 90, 062805 (2014).

[8] T. P. Peixoto, Inferring the Mesoscale Structure of Layered, Edge-Valued, and Time-Varying Networks, Phys. Rev. E 92, 042807 (2015).

[9] N. Stanley, S. Shai, D. Taylor, and P. J. Mucha, Clustering Network Layers with the Strata Multilayer Stochastic Block Model, arXiv:1507.01826.

[10] C. Aicher, A. Z. Jacobs, and A. Clauset, Learning Latent Block Structure in Weighted Networks, J. Complex Netw. 3, 221 (2015).

[11] T. Vallés-Catalè, F. A. Massucci, R. Guimerà, and M. SalesPardo, Multilayer Stochastic Block Models Reveal the Multilayer Structure of Complex Networks, Phys. Rev. X 6, 011036 (2016).

[12] C. Moore, X. Yan, Y. Zhu, J.-B. Rouquier, and T. Lane, in Proceedings of the 17th ACM SIGKDD International Conference on Knowledge Discovery and Data Mining, KDD '11, (publisher ACM, New York, 2011), pp. 841-849.

[13] M. Leng, Y. Yao, J. Cheng, W. Lv, and X. Chen, Active SemiSupervised Community Detection Algorithm with Label Propagation, Lect. Notes Comput. Sci. 7826, 324 (2013).

[14] L. Peel, Active Discovery of Network Roles for Predicting the Classes of Network Nodes, J. Complex Netw. 3, 431 (2015).

[15] J. Yang, J. McAuley, and J. Leskovec, in Proceedings of the 2013 IEEE 13th International Conference on Data Mining (ICDM) (IEEE, New York, 2013), pp. 1151-1156.

[16] C. Bothorel, J. D. Cruz, M. Magnani, and B. Micenkova, Clustering Attributed Graphs: Models, Measures and Methods, arXiv:1501.01676 [Netw. Sci. J. (to be published)]. 
[17] P. Zhang, C. Moore, and L. Zdeborovà, Phase Transitions in Semisupervised Clustering of Sparse Networks, Phys. Rev. E 90, 052802 (2014).

[18] Z.-Y. Zhang, Community Structure Detection in Complex Networks with Partial Background Information, Europhys. Lett. 101, 48005 (2013).

[19] A. Freno, G. Garriga, and M. Keller, Neural Information Processing Systems Workshop on Choice Models and Preference Learning, Granada, Spain, 2011, https://hal .inria.fr/hal-00641419.

[20] M. E. J. Newman and A. Clauset, Structure and Inference in Annotated Networks, Nat. Commun. 7, 11863 (2016).

[21] D. Liben-Nowell and J. Kleinberg, The Link-Prediction Problem for Social Networks, J. Am. Soc. Inf. Sci. Technol. 58, 1019 (2007).

[22] A. Clauset, C. Moore, and M. E. J. Newman, Hierarchical Structure and the Prediction of Missing Links in Networks, Nature (London) 453, 98 (2008).

[23] R. Guimerà and M. Sales-Pardo, Missing and Spurious Interactions and the Reconstruction of Complex Networks, Proc. Natl. Acad. Sci. U.S.A. 106, 22073 (2009).

[24] R. Guimerà and M. Sales-Pardo, A Network Inference Method for Large-Scale Unsupervised Identification of Novel Drug-Drug Interactions, PLoS Comput. Biol. 9, e1003374 (2013).

[25] N. Rovira-Asenjo, T. Gumí, M. Sales-Pardo, and R. Guimerà, Predicting Future Conflict between TeamMembers with Parameter-Free Models of Social Networks, Sci. Rep. 3, 1999 (2013).

[26] N. Musmeci, S. Battiston, G. Caldarelli, M. Puliga, and A. Gabrielli, Bootstrapping Topological Properties and Systemic Risk of Complex Networks Using the Fitness Model, J. Stat. Phys. 151, 720 (2013).

[27] G. Cimini, T. Squartini, A. Gabrielli, and D. Garlaschelli, Estimating Topological Properties of Weighted Networks from Limited Information, Phys. Rev. E 92, 040802 (2015).

[28] R. A. Rossi, L. K. McDowell, D. W. Aha, and J. Neville, Transforming Graph Data for Statistical Relational Learning, J. Artif. Intell. Res. 45, 363 (2012).

[29] B. Bringmann, M. Berlingerio, F. Bonchi, and A. Gionis, Learning and Predicting the Evolution of Social Networks, IEEE Intell. Syst. 25, 26 (2010).

[30] M. Kim and J. Leskovec, in Proceedings of the 2011 SIAM International Conference on Data Mining (Society for Industrial and Applied Mathematics, Philadelphia, 2011), pp. 47-58.

[31] M. Kivelä, A. Arenas, M. Barthelemy, J. P. Gleeson, Y. Moreno, and M. A. Porter, Multilayer Networks, J. Complex Netw. 2, 203 (2014).

[32] M. De Domenico, A. Solé-Ribalta, E. Cozzo, M. Kivelä, Y. Moreno, M. A. Porter, S. Gómez, and A. Arenas, Mathematical Formulation of Multilayer Networks, Phys. Rev. X 3, 041022 (2013).

[33] T. P. Peixoto, Hierarchical Block Structures and HighResolution Model Selection in Large Networks, Phys. Rev. X 4, 011047 (2014).

[34] B. Karrer and M. E. J. Newman, Stochastic Blockmodels and Community Structure in Networks, Phys. Rev. E 83, 016107 (2011).
[35] T. P. Peixoto, Entropy of Stochastic Blockmodel Ensembles, Phys. Rev. E 85, 056122 (2012).

[36] T. P. Peixoto, Model Selection and Hypothesis Testing for Large-Scale Network Models with Overlapping Groups, Phys. Rev. X 5, 011033 (2015).

[37] P. D. Grünwald, The Minimum Description Length Principle (The MIT Press, Cambridge, MA, 2007).

[38] M. Rosvall and C. T. Bergstrom, An Information-Theoretic Framework for Resolving Community Structure in Complex Networks, Proc. Natl. Acad. Sci. U.S.A. 104, 7327 (2007).

[39] E. T. Jaynes, in Probability Theory: The Logic of Science, edited by G. Larry Bretthorst (Cambridge University Press, Cambridge, England, 2003).

[40] T. P. Peixoto, Parsimonious Module Inference in Large Networks, Phys. Rev. Lett. 110, 148701 (2013).

[41] More precisely, the approach of Ref. [20] is based on semiBayesian inference, where priors for only part of the parameters are specified (the node partition) but not others (the metadata-group and group-group affinities, as well as node degrees). This approach is less susceptible to overfitting when compared to pure maximum likelihood, but cannot be used to select the model order (via the number of groups) as we do here, for the reasons explained in the text (see also Ref. [42]).

[42] A. Decelle, F. Krzakala, C. Moore, and L. Zdeborová, Asymptotic Analysis of the Stochastic Block Model for Modular Networks and Its Algorithmic Applications, Phys. Rev. E 84, 066106 (2011).

[43] T. P. Peixoto, Efficient Monte Carlo and Greedy Heuristic for the Inference of Stochastic Block Models, Phys. Rev. E 89, 012804 (2014).

[44] T.P. Peixoto, The Graph-Tool Python Library, https:// figshare.com/articles/graph-tool/1164194; see also http:// graph-tool.skewed.de.

[45] M. Girvan and M. E. J. Newman, Community Structure in Social and Biological Networks, Proc. Natl. Acad. Sci. U.S.A. 99, 7821 (2002).

[46] T. S. Evans, Clique Graphs and Overlapping Communities, J. Stat. Mech. (2010) P12037.

[47] T S. Evans, American College Football Network Files, https://figshare.com/articles/ American-College-Football-Network-Files/93179.

[48] R. Guimerà, M. Sales-Pardo, and L. A. N. Amaral, Modularity from Fluctuations in Random Graphs and Complex Networks, Phys. Rev. E 70, 025101 (2004).

[49] B. H. Good, Y.-A. de Montjoye, and A. Clauset, Performance of Modularity Maximization in Practical Contexts, Phys. Rev. E 81, 046106 (2010).

[50] A. Lancichinetti, S. Fortunato, and F. Radicchi, Benchmark Graphs for Testing Community Detection Algorithms, Phys. Rev. E 78, 046110 (2008).

[51] See https://sites.google.com/site/santofortunato/ inthepress2.

[52] Retrieved from http://geneontology.org/.

[53] S. R. Collins, P. Kemmeren, X.-C. Zhao, J. F. Greenblatt, F. Spencer, F. C. P. Holstege, J.S. Weissman, and N. J. Krogan, Toward a Comprehensive Atlas of the Physical Interactome of Saccharomyces cerevisiae, Mol. Cell Proteomics 6, 439 (2007). 
[54] H. Yu et al., High-Quality Binary Protein Interaction Map of the Yeast Interactome Network, Science 322, 104 (2008).

[55] D. Park, R. Singh, M. Baym, C.-S. Liao, and B. Berger, IsoBase: A Database of Functionally Related Proteins across PPI Networks, Nucleic Acids Res. 39, D295 (2011).

[56] Q. C. Zhang, D. Petrey, J. I. Garzón, L. Deng, and B. Honig, PrePPI: A Structure-Informed Database of Protein-Protein Interactions, Nucleic Acids Res. 41, D828 (2013).

[57] A. L. Traud, P. J. Mucha, and M. A. Porter, Social Structure of Facebook Networks, Physica (Amsterdam) 391A, 4165 (2012).

[58] See www.caida.org/.

[59] L. Backstrom, D. Huttenlocher, J. Kleinberg, and X. Lan, in Proceedings of the 12th ACM SIGKDD International Conference on Knowledge Discovery and Data Mining, KDD 'O6 (ACM, New York, 2006), pp. 44-54.

[60] Retrieved from http://snap.stanford.edu/data/com-DBLP .html.

[61] L. M. Aiello, M. Deplano, R. Schifanella, and G. Ruffo, in Proceedings of the 6th International AAAI Conference on Weblogs and Social Media, Dublin, IR, 2012 (AAAI Press, Palo Alto, California, 2012), https://www.aaai.org/Library/ ICWSM/icwsm12contents.php.

[62] L. M. Aiello, A. Barrat, C. Cattuto, G. Ruffo, and R. Schifanella, in Proceedings of the 2010 IEEE Second
International Conference on Social Computing (SocialCom) (IEEE, New York, 2010), pp. 249-256.

[63] O. Richters and T. P. Peixoto, Trust Transitivity in Social Networks, PLoS One 6, e18384 (2011).

[64] A. Mislove, M. Marcon, K. P. Gummadi, P. Druschel, and B. Bhattacharjee, in Proceedings of the 7th ACM SIGCOMM Conference on Internet Measurement, IMC '07, (ACM, New York, 2007), pp. 29-42.

[65] L. A. Adamic and N. Glance, in Proceedings of the 3rd International Workshop on Link Discovery, LinkDD '05, (ACM, New York, 2005), pp. 36-43.

[66] See http://www.debian.org.

[67] See https://wiki.debian.org/Debtags.

[68] E. Zini, in Proceedings of the 5th Annual Debian Conference, 2005, pp. 59-74, http://debtags.alioth.debian .org/paper-debtags.pdf.

[69] See *_Packages files in the /var/lib/apt/ directory in an installed system or on mirrors, for example, ftp://ftp.debian .org/debian/dists/wheezy/main/binary-amd64/.

[70] J. Leskovec, L. A. Adamic, and B. A. Huberman, The Dynamics of Viral Marketing, ACM Trans. Web 1, 5 (2007).

[71] See http://snap.stanford.edu/data/amazon-meta.html.

[72] See http://www.imdb.com.

[73] Retrieved from http://publish.aps.org/dataset. 